\title{
The use of laser capture microscopy in proteomics research - A review
}

\author{
George M. Kunz Jr. and Daniel W. Chan \\ Department of Pathology, Division of Clinical Chemistry, The Johns Hopkins University School of Medicine, 600 \\ North Wolfe Street, Meyer B-121, Baltimore, Maryland 21205, USA \\ Tel.: +1 410955 6304; Fax: +1 410614 7609; E-mail: gkunz@jhmi.edu,dchan@jhmi.edu
}

\section{Introduction}

The sequencing of the human genome began with much fanfare in 1990 with the start of the Human Genome Project. Major portions of that program have been completed since the interim draft of the human sequence was published in 2001 [1]. Scientists now turn their attention to an even more complex and arduous task, characterizing the human 'proteome'. The term proteome was coined to describe all the proteins present in a cell, tissue, or an organism [2]. The human genome consists of roughly three billion base pairs of deoxyribonucleic acid, encoding about 30,000 genes. By means of alternative splicing of messenger RNA, these genes are thought to encode over 100,000 proteins. Yet this number only hints at the complexity of the human proteome, for each protein is modified posttranslationally, is expressed in differing amounts in different tissues under different conditions, and interacts in broad networks with other proteins to regulate the physiology of the cell and the organism overall. Thus, characterizing all these proteins and deducing their role, which is the goal of the science of 'proteomics', will require a number of new and innovative tools [3].

The study of proteins is not new. In fact, the field of biochemistry was built on the study of proteins functioning as enzymes. Tools from that era are constantly being adapted to further our understanding of proteins during the 'proteomic' era. The tools can be used alone or in combination with one another to separate, purify, and ultimately identify proteins. For instance, twodimensional gel electrophoresis (2DE) can separate a complex mixture of proteins into its constituent proteins based on their size and charge. From 3000 to 10,000 proteins can be separated into spots on a gel depending on the sensitivity of the spot detection method that is used [4]. Mass spectrometry can be used to further characterize the separated proteins, even identify specific proteins sequences based on the mass spectrum produced. Newer techniques such as surface-enhanced laser desorption/ionization time-of-flight mass spectrometry (SELDI TOF MS) are building on older technologies to create high-throughput methods to characterize proteins in serum or in a cell [5]. A new technology that is increasingly being employed in proteomics research is laser capture microscopy.

\section{Laser capture microscopy}

Laser capture microscopy (LCM) was developed to deal with the problem of tissue heterogeneity. By definition, a tissue is a mixture of different cell types. For example, in the normal liver, there are not only hepatocytes but also small blood vessels containing endothelial cells and smooth muscle cells, Kupffer cells of the reticuloendothelial system, and bile duct epithelial cells, to name just a few. Even in a tumor, which one would assume is a pure monoclonal population of cells, there are other cells intermingled with the tumor cells including fibroblasts, endothelial cells, and inflammatory cells. Flow cytometry is an example of a technology that allowed purification of populations of lymphocytes from the blood. Unfortunately, obtaining pure 
populations of cells from tissue is more difficult since these cells are not suspended in a free flowing medium. A number of different techniques, however, have been developed to obtain a pure population of cells in tissues. This pure population is a cleaner starting point for today's genomic and proteomic technologies, which have become so sensitive that contaminating cells can confound experimental data.

A crude technique to obtain purer populations of tumor cells is gross dissection, i.e. physically separating a tumor mass from normal tissue with the aid of a scalpel and the naked eye [6]. Refinements to this technique include adding a microscope to more finely delineate and separate tumor from normal tissue or using a laser to ablate the surrounding normal tissue $[7,8]$. However, the resulting tissue is still not pure enough since these techniques do not deal with the problem of heterogeneity within the tumor itself. Researchers at the National Institutes of Health $(\mathrm{NIH})$ tackled this problem by developing laser capture microscopy (LCM), which was commercialized in 1997 by Arcturus Engineering (Mountain View, California) through a Collaborative Research and Development Agreement (CRADA) [911]. The current instruments are called PixCell II and AutoPix [9].

The technique of LCM is quite ingenious (see Figure). Tissue sections are cut from a paraffin microtome or cryostat to a thickness of between five and ten microns and placed on a slide. After a standard series of washes, the slide is stained with hematoxylin and eosin (H\&E) or an immunohistochemical stain. A thin sterile thermoplastic film of ethylene vinyl acetate (EVA) polymer is then placed over the tissue section on the slide. The tissue is visualized with an inverted microscope, which allows for localization of the cell of interest. A near-infrared laser is fired at the cell, fusing it to the film. The film is removed with the cell of interest, whose contents can be extracted from the film using standard lysis buffers. The lysate is analyzed by a variety of methods including 2DE and mass spectrometry [9-12].

The beauty of this technique is that more than one cell can be collected at a time. Multiple histologically similar cells of a similar lineage can be collected on one thermoplastic film. Larger structures, such as whole glomeruli from kidney, can also be captured. The 50milliwatt laser fires a 50-millisecond pulse that is $30 \mathrm{mi}-$ crons in diameter (for reference, a red blood cell has a diameter of about 7 microns). With one slide, an experienced microscopist can fire between 1000 and 3000 laser shots to capture at least 6000 cells in about 20
The Laser Capture Microdissection Process

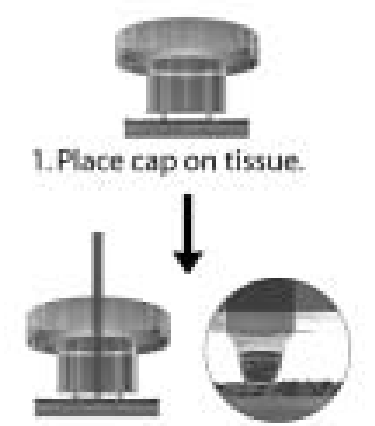

2. Pulse laser on target cells.

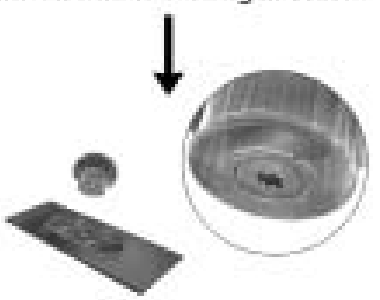

3. Remove cap with adhered target cells.

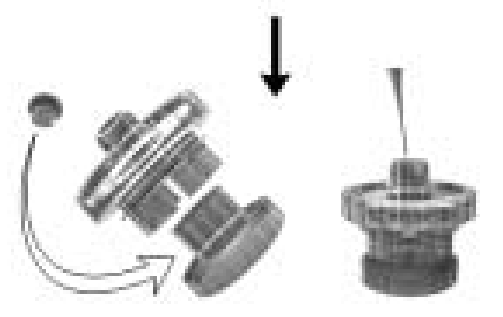

4. Extract molecules from target cells.

Fig. 1. Graphic summarizing the laser capture microdissection process (used with permission of Arcturus Engineering, Mountain View, CA).

minutes $[9,10]$. Further refinements to LCM have been made. For instance, the EVA film has been attached to the bottom of the cap of a $0.5 \mathrm{~mL}$ Eppendorf tube to ease the transfer of the cells to lysis buffer. Suarez et al also describe a modification to this system called "cylinder-based LCM" (cLCM). Instead of using a flat sheet of thermoplastic film to capture cells, a cone of film is used. The cone is attached to the manipulators of the stage of the microscope and can be rotated as the slide is moved. The cone can be lifted off one slide while another slide is put in its place. In this way, additional cells can be collected from multiple different tissue sections on different slides $[9,10]$. 


\section{Advantages of LCM}

There are a number of advantages to this system. The components of the system are fairly low cost [6]. Also, the number and types of cells and structures can be easily documented and counted by examining the film under a digital microscope $[6,11]$. The presence of contaminating cells can also be assessed [9]. In addition to normal and malignant cells, dysplastic cells or nonneoplastic cells from the same tissue can be selectively purified and compared with the benign and malignant cell [11]. In addition, at least with regard to analysis of DNA and RNA, a number of different archival tissue specimens can be used including formalin-fixed paraffin-embedded tissue and cytologic specimens. Finally, the tissues can be stained by conventional H\&E or by immunohistochemistry to identify the particular cells of interest.

The film and heat produced by the low power laser do not affect analysis of DNA, RNA, or protein. This was confirmed by Banks et al who compared tissue collected by LCM with tissue collected by more conventional methods. They found no gross effects on protein profiles between LCM-collected and conventionallycollected tissue when compared by electrophoresis. Two tested proteins, HSP-60 and $\beta 2$-microglobulin, retained their antigenicity on Western blot [7].

\section{Disadvantages of LCM}

There are a number of technical limitations to LCM at least in regard to the study of proteins. First, LCM for use in proteomics research requires fresh frozen tissue. Formalin-fixed tissue cannot be used to study proteins since formalin extensively crosslinks proteins. Extraction protocols to use with Western blots have been developed for formalin-fixed paraffin-embedded tissue [13]. However, these probably would not be applicable to biomarker discovery using 2DE and mass spectrometry, which require more native structure to be retained in extracted proteins [13]. It is possible to use ethanol-fixed paraffin embedded tissue in some proteomics applications such as 2DE [14]. Unfortunately, most archival tissue specimens in pathology departments use formalin, requiring researchers to still collect tissue fresh at the time of surgery.

Second, many dyes used routinely to stain tissue can interfere with proteomic tools such as 2DE. Craven et al states that the detrimental effect of these dyes has less to do with the dye itself but more to do with relative incubation time of slides in aqueous solutions (which differs for each dye), and with the intensity of staining [15]. Eosin, in particular, interferes with protein migration on 2DE [14]. Fortunately, hematoxylin and methyl green seem to have no effect on protein migration so they could potentially be used alone to stain tissue for LCM [15]. Another technique to overcome this problem is described by Mouledous et al. who used "navigated LCM" to dissect rat brain nuclei. This technique involves staining one slide level with $\mathrm{H} \& \mathrm{E}$, capturing the slide image digitally, and using that image to capture cells on an adjacent unstained slide [16].

Finally, LCM can be time consuming. When the tissue of interest contains a number of different intermingled cells types, time must be spent dissecting out the cells of interest. Marko-Varga et al captured 7200 hepatocytes in 20 minutes in a relatively homogeneous sample of liver after 6000 laser shots using an automated dissection procedure [17]. However, it took Banks et al. 13 hours to microdissect enough epithelial cells from the uterine cervix to use on a $2 \mathrm{DE}$ gel. They note that LCM will probably never be used for routine diagnostics [7]. Also, unless ultrasensitive detection methods for proteins are used, thousands of cells must be collected to get enough protein to run on a $2 \mathrm{DE}$ gel. Fortunately, surface-enhanced laser desorption/ionization time-of-flight mass spectrometry (SELDI TOF MS) using ProteinChip technology (Ciphergen, Fremont, California) is a new technique that is very sensitive for protein and thus particularly useful in this setting. It uses chips with chromatographic surfaces to separate small amounts of proteins based on multiple chemical properties such as charge and then analyzes the protein with TOF MS. It is sensitive for proteins to femtomole or attomole concentrations and can produce a protein profile from as few as 25 cells, although 500 to 1000 cells should be used for reproducibility $[8,18$, 19]. At this level of sensitivity, SELDI TOF MS produces a protein profile of the collected cells based on mass-to-charge $(\mathrm{m} / \mathrm{z})$ ratio. To identify the protein of interest, additional steps are required. These include purification of the protein based on the type of chromatographic chip used to produce the initial protein profile and then identification of the purified protein by peptide mapping and tandem MS [20].

\section{Examples of LCM in proteomics research}

The strength of LCM is the ability to purify normal and cancerous cells from the same specimen and an- 
alyze them with various proteomic techniques. Early papers in the medical literature mostly concentrated on the feasibility of using LCM with 2DE and mass spectrometry. More recent papers have identified proteins that are differentially expressed in benign and malignant cells and thus could potentially be used as new diagnostic biomarkers or targets for therapy. Some of these papers are summarized below.

In 2000 Palmer-Toy et al published a paper showing the feasibility of analyzing a lysate of captured cells with matrix-assisted laser desorption/ionization time-of-flight mass spectrometry (MALDI-TOF) without intervening separation or purification steps such as 2DE [21]. They took frozen sections from one modified radical mastectomy specimen, stained with H\&E, and captured 2500 normal breast epithelial cells, normal stromal cells, in situ carcinoma cells, invasive carcinoma cells, and metastatic cells to a lymph node. The captured cells were estimated to be $>98 \%$ homogeneous. The lysate from these cells were analyzed by MALDI-TOF and the peaks produced by the different cell types were compared. Several high mass peaks were identified in the $45-60 \mathrm{kDa}$ range that were different in malignant versus benign epithelial cells and between in situ carcinoma cells and benign epithelial cells. The mass spectra were identical for metastatic and invasive tumors. All spectra were reproducible as well. They did not go on to identify the proteins that produced the peaks because not enough protein was available for determination of protein composition by mass spectra alone. A similar study using SELDI-TOF found that a combination of mass spectra peaks might be used to discriminate between benign and malignant cells in the prostate [22].

Another interesting application of LCM was demonstrated by Simone et al. [23,24]. They used LCM to microdissect populations of normal prostate epithelial cells and cells from prostate intraepithelial neoplasia and invasive prostate adenocarcinoma. They then used an automated sandwich chemiluminescent immunoassay to calculate the number of prostate specific antigen (PSA) molecules per cell. They confirmed what had only been demonstrated qualitatively by immunohistochemical stains, namely, that PSA expression is heterogeneous in prostate adenocarcinoma, i.e. it varies considerably from carcinoma cell to carcinoma cell within the same tumor. Normal prostate epithelium, by contrast, had a more constant PSA content from cell to cell. The authors suggested that cellular quantitation of HER-2/neu might be a future application for this technique $[23,24]$. Heterogeneity of protein profiles between carcinoma cells of a single tumor was confirmed in other studies, including one of infiltrating duct carcinoma of the breast [25].

Ornstein et al. have written a number of papers using LCM in proteomics studies. In one study, they dissected normal and malignant epithelial cells and benign stromal cells from frozen sections of three radical prostatectomy specimens [26]. Lysates from 40,000 to 50,000 cells were run on $2 \mathrm{DE}$ gels producing about 750 protein spots. Six of the proteins were found at detectable levels only in malignant epithelial cells while two were found only in normal cells. One of these proteins, prostate specific antigen (PSA), was found only in malignant cells. As a control, benign prostate stromal cells were microdissected and compared with benign and malignant epithelial cells. Fewer than $45 \%$ of proteins were shared between the epithelial and stromal cells. They also compared 2DE gels from lysates of two metastatic prostate cancer cell lines ( $\mathrm{LnCaP}$ and PC3). Fewer than $20 \%$ of proteins from the cultured cells were shared with the benign and malignant epithelial cells from the radical prostatectomies, raising the question of whether cultured tumors cells are an appropriate substitute for studying carcinoma in vivo [26]. In another study, Ornstein et al used LCM-dissected cells to determine if intracellular PSA is bound or unbound in normal and malignant cells [27]. An answer to this might provide an explanation as to why patients with lower percentages of free serum PSA are more likely to have cancer than patients with higher percentages, at least in the diagnostic gray-zone of total serum PSA (4-10 ng/mL). They found that PSA was present in unbound form in both normal and malignant prostate epithelial cells. The explanation as to why patients with prostate carcinoma are more likely to have a lower percentage of free PSA in serum remained unexplained.

In another study, Zheng et al. found a putative marker for prostate cancer, which they named $\mathrm{PCa}-24$, by comparing the mass spectra from whole sections of benign and tumor tissue using SELDI TOF MS. To confirm that the putative marker was indeed produced by the malignant epithelial cells, they microdissected benign and malignant epithelial cells, showing the putative marker was found only in the malignant epithelial cells and not in the benign cells [19].

Other tumors, on which LCM has been used in proteomics research, include esophageal carcinoma. Pawleletz et al used LCM and Western blotting to show that there was a complete loss or reduced expression of annexin I in esophageal squamous cell carcinoma and prostate adenocarcinoma when compared with nor- 
mal epithelium and stromal cells that were microdissected as controls [28]. They also showed that this reduced expression was present in adjacent dysplastic (i.e. premalignant) epithelium. A collaborator of this group published a similar report using LCM and 2DE on esophageal tumors showing differential expression of additional proteins in tumor versus normal cells [29]. One of these proteins was annexin I while another was cytokeratin 1 .

Finally, Melle et al. used LCM to enrich cells from head and neck squamous cell carcinomas. Compared with normal epithelial cells, the carcinoma cells showed a significant difference in one mass spectrum peak. This was identified as annexin $\mathrm{V}$ by proteolytic digestion of a spot on a 2DE gel and peptide mapping by tandem mass spectrometry [18].

\section{Conclusion}

The field of proteomics is growing rapidly as new methods for high-throughput protein analysis are developed and exploited. One of the strengths of highthroughput methods such as SELDI TOF MS is the ability to compare protein profiles in complex samples such as serum and tissue from cancer and non-cancer patients. The profile can then be used to identify proteins unique to the cancerous sample, thus providing a target for research efforts to enhance diagnosis and therapy. However, even though SELDI TOF MS can pick up extremely low levels of proteins, it still may not be sensitive enough to identify a unique protein due to confounding factors. Some of these factors include the sheer numbers of higher abundant proteins present in a clinical specimen such as serum and whether a putative biomarker protein is elaborated by the tumor itself or is present for another reason [30]. Laser capture microscopy helps eliminate these variables. It literally allows a researcher to compare benign and malignant cells from the same tissue in the same patient. The cells can then be compared by today's mass throughput technologies. Future directions for LCM include analyzing many different tumors for new diagnostic biomarkers and therapeutic targets. In addition, it may be used in initiatives such as the Cancer Genome Anatomy Project (cgap.nci.nih.gov) to catalog the protein profiles of normal, precancerous, and cancerous cells [9].

\section{References}

[1] D. Baltimore, Our genome unveiled, Nature 409 (2001), 814 816 .
[2] T. Werner, Proteomics and regulomics: the yin and yang of functional genomics, Mass Spectrom Rev 23 (2004), 25-33.

[3] S.D. Patterson and R.H. Aebersold, Proteomics: the first decade and beyond, Nat Genet 33 (2003), 311-323.

[4] W. Clarke and Z. Zhang et al., The application of clinical proteomics to cancer and other diseases, Clin Chem Lab Med 41 (2003), 1562-1570.

[5] R. Aebersold and M. Mann, Mass spectrometry-based proteomics, Nature 422 (2003), 198-207.

[6] M.R. Emmert-Buck and R.F. Bonner et al., Laser capture microdissection, Science 274 (1996), 998-1001.

[7] R.E. Banks and M.J. Dunn et al., The potential use of laser capture microdissection to selectively obtain distinct populations of cells for proteomic analysis-preliminary findings, Electrophoresis 20 (1999), 689-700.

[8] R.A. Craven and R.E. Banks, Laser capture microdissection and proteomics: possibilities and limitation, Proteomics 1 (2001), 1200-1204.

[9] C.A. Suarez-Quian and S.R. Goldstein et al., Laser capture microdissection: a new tool for the study of spermatogenesis, $J$ Androl 21 (2000), 601-608.

[10] C.A. Suarez-Quian and S.R. Goldstein et al., Laser capture microdissection of single cells from complex tissues, Biotechniques 26 (1999), 328-335.

[11] R.F. Bonner and M. Emmert-Buck et al., Laser capture microdissection: molecular analysis of tissue, Science $\mathbf{2 7 8}$ (1997), 1481,83.

[12] D.E. Palmer-Toy, S. Kuzdzal and D.W. Chan, Proteomic approaches to tumor marker discovery, in: Tumor markers: physiology, pathobiology, technology, and clinical applications, E.P. Diamandis et al, ed., AACC Press, Washington, D.C., 2002, pp. 391-400.

[13] K. Ikeda and T. Monden et al., Extraction and analysis of diagnostically useful proteins from formalin-fixed, paraffinembedded tissue sections, J Histochem Cytochem 46 (1998), 397-403.

[14] M. Ahram and M.J. Flaig et al., Evaluation of ethanol-fixed, paraffin-embedded tissues for proteomic applications, Proteomics 3 (2003), 413-421.

[15] R.A. Craven and N. Totty et al., Laser capture microdissection and two-dimensional polyacrylamide gel electrophoresis: evaluation of tissue preparation and sample limitations, $A m \mathrm{~J}$ Pathol 160 (2002), 815-822.

[16] L. Mouledous and S. Hunt et al., Navigated laser capture microdissection as an alternative to direct histological staining for proteomic analysis of brain samples, Proteomics 3 (2003), 610-615.

[17] G. Marko-Varga and M. Berglund et al., Targeting hepatocytes from liver tissue by laser capture microdissection and proteomics expression profiling, Electrophoresis 24 (2003), 3800-3805.

[18] C. Melle and G. Ernst et al., Biomarker Discovery and Identification in Laser Microdissected Head and Neck Squamous Cell Carcinoma with ProteinChip(R) Technology, Twodimensional Gel Electrophoresis, Tandem Mass Spectrometry, and Immunohistochemistry, Mol Cell Proteomics 2 (2003), 443-452.

[19] Y.Zheng and Y. Xu et al., Prostate carcinoma tissue proteomics for biomarker discovery, Cancer 98 (2003), 2576-2582.

[20] H.J. Issaq and T.D. Veenstra et al., The SELDI-TOF MS approach to proteomics: protein profiling and biomarker identification, Biochem Biophys Res Commun 292 (2002), 587-592.

[21] D.E. Palmer-Toy and D.A. Sarracino et al., Direct acquisition of matrix-assisted laser Desorption/Ionization time-of-flight 
mass spectra from laser capture microdissected tissues, Clin Chem 46 (2000), 1513-1516.

[22] L.H. Cazares and B.L. Adam et al., Normal, benign, preneoplastic, and malignant prostate cells have distinct protein expression profiles resolved by surface enhanced laser desorption/ionization mass spectrometry, Clin Cancer Res 8 (2002), 2541-2552.

[23] N.L. Simone and A.T. Remaley et al., Sensitive immunoassay of tissue cell proteins procured by laser capture microdissection, Am J Pathol 156 (2000), 445-452.

[24] N.L. Simone and C.P. Paweletz et al., Laser capture microdissection: beyond functional genomics to proteomics, $\mathrm{Mol} \mathrm{Di}$ agn 5 (2000), 301-307.

[25] J.D. Wulfkuhle and K.C. McLean et al., New approaches to proteomic analysis of breast cancer, Proteomics 1 (2001), 1205-1215.

[26] D.K. Ornstein and J.W. Gillespie et al., Proteomic analysis of laser capture microdissected human prostate cancer and in vitro prostate cell lines, Electrophoresis 21 (2000), 22352242.

[27] D.K. Ornstein and C. Englert et al., Characterization of intracellular prostate-specific antigen from laser capture microdissected benign and malignant prostatic epithelium, Clin Cancer Res 6 (2000), 353-356.

[28] C.P. Paweletz and D.K. Ornstein et al., Loss of annexin 1 correlates with early onset of tumorigenesis in esophageal and prostate carcinoma, Cancer Res 60 (2000), 6293-6297.

[29] M.R. Emmert-Buck and J.W. Gillespie et al., An approach to proteomic analysis of human tumors, Mol Carcinog 27 (2000), $158-165$.

[30] E.P. Diamandis, Mass spectrometry as a diagnostic and a cancer biomarker discovery tool: Opportunities and potential limitations, Mol Cell Proteomics (2004). 


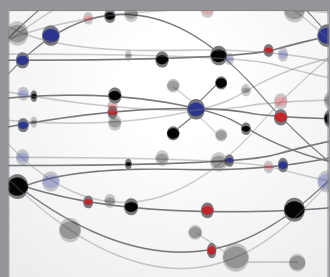

The Scientific World Journal
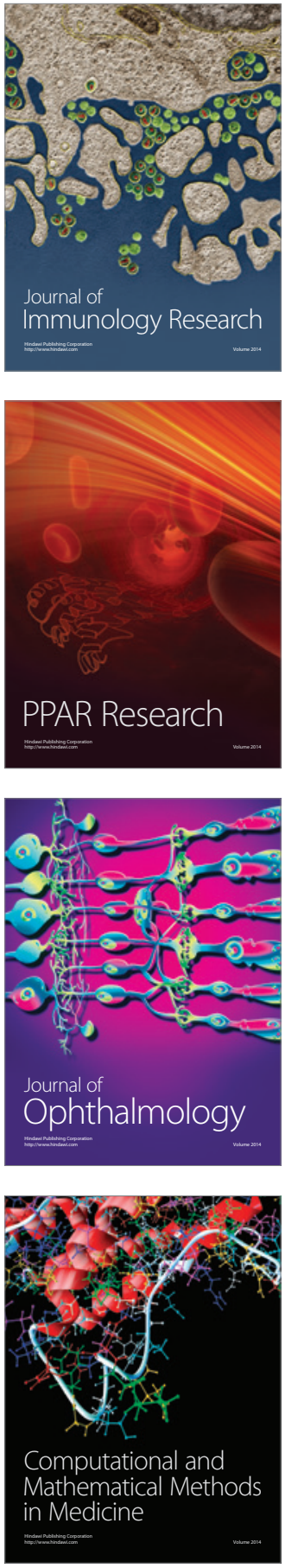

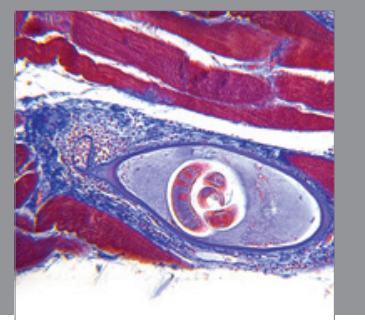

Gastroenterology

Research and Practice
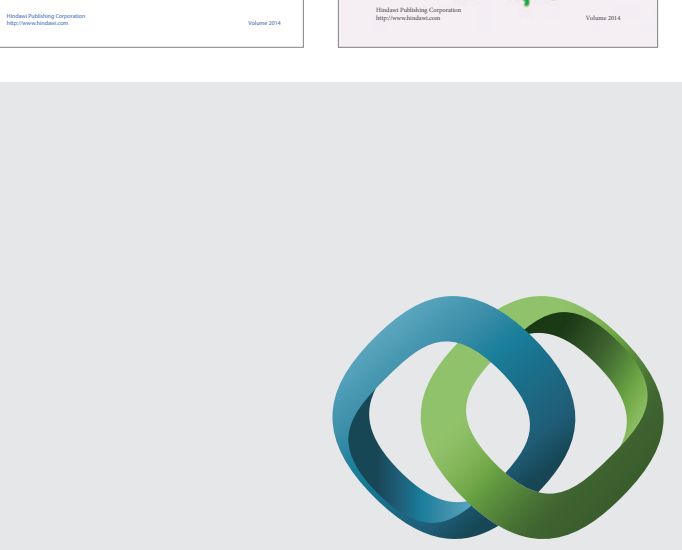

\section{Hindawi}

Submit your manuscripts at

http://www.hindawi.com
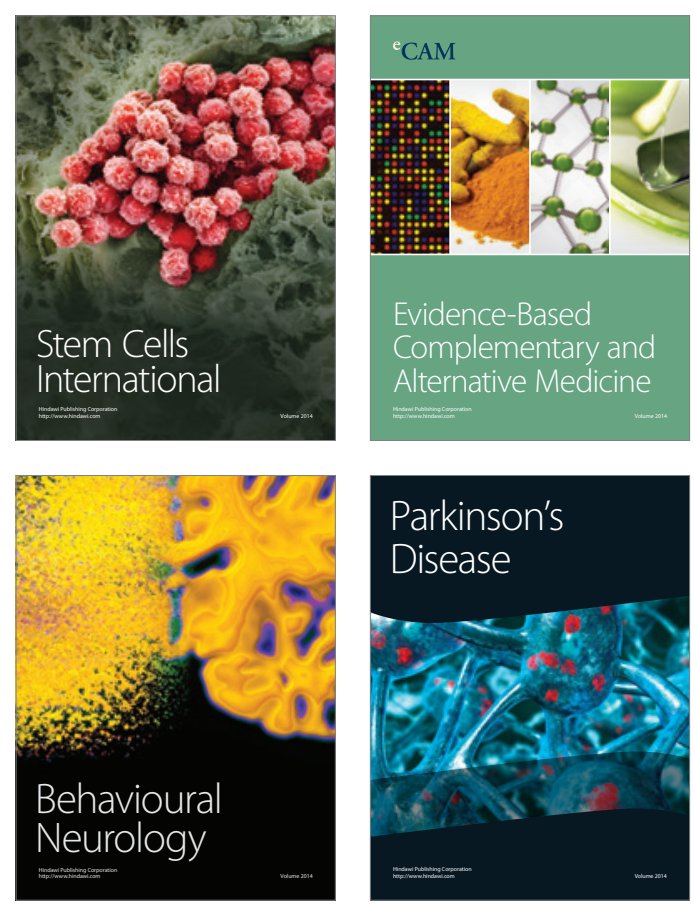

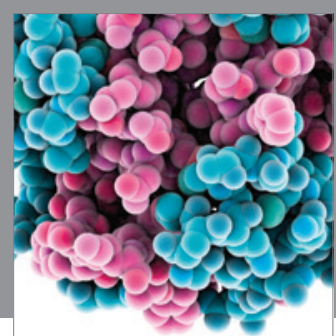

Journal of
Diabetes Research

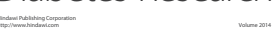

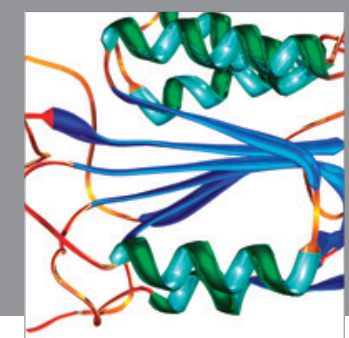

Disease Markers
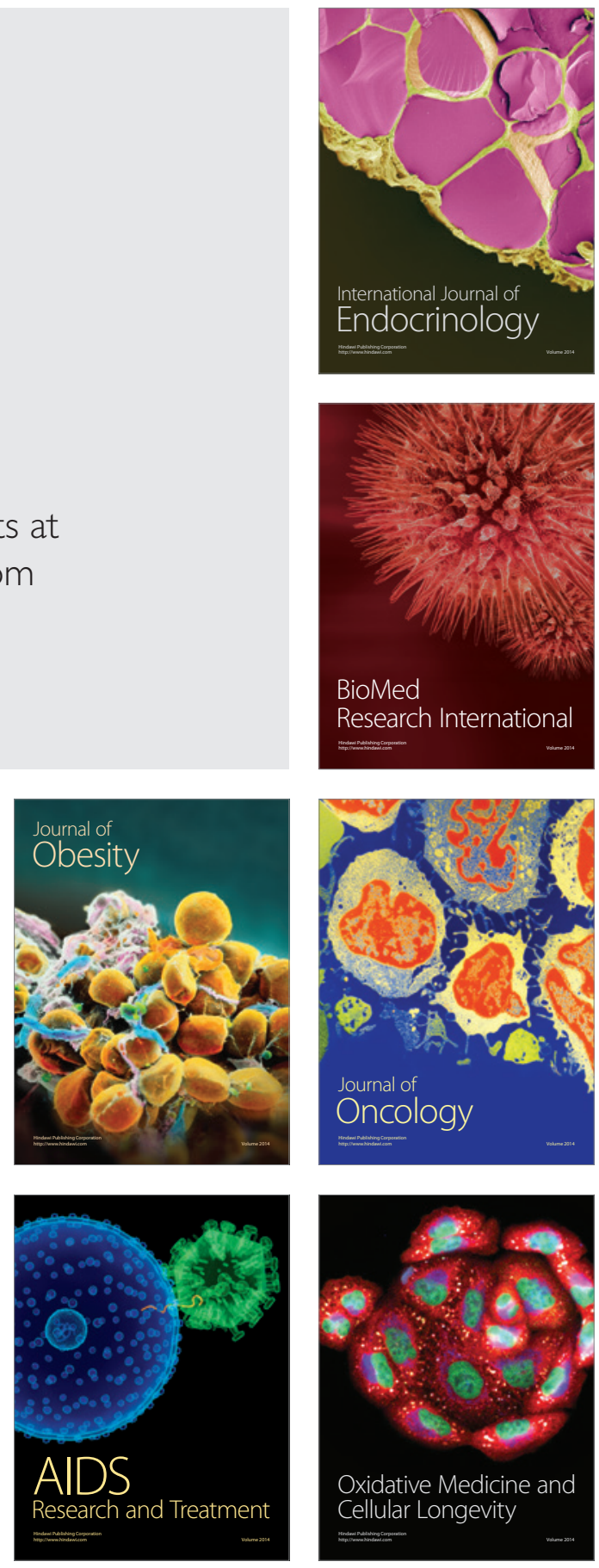